\title{
Link Conflict in Multistage Interconnection Networks
}

\author{
Shobha Arya*, Nipur Singh** \\ *(Department of Computer Science, Gurukul Kangri Vishwavidyalaya, Haridwar \\ ** (Department of Computer Science, Gurukul Kangri Vishwavidyalaya, Haridwar
}

\begin{abstract}
Multistage interconnection networks (MINs) are high performance indispensable networks with low cost for broadband switching technology and multiprocessor systems. Crosstalk is a critical issue in MINs. The undesired coupling of two or more wavelengths is reason behind Crosstalk and Link Conflicts and Switch Conflicts can cause it. In Electrical or traditional MINs, link conflict is the only reason behind crosstalk but in optical MINs, both conflicts (switch conflicts and link conflicts) occurs in crosstalk. In traditional MIN or electrical MIN, the link conflict problem can also be arises because of congestion. In this paper, we are going to discuss about link conflict problem in electrical MIN. In this paper, we proposed a new link conflict detection method named Link Window Method (LWM) to detect link conflicts in omega MIN. Congestion can also be identified in switches with the help of proposed algorithm.
\end{abstract}

Keywords: Crosstalk; Link Conflict; Multistage Interconnection Networks

\section{INTRODUCTION}

Multistage Interconnection networks (MINs) plays a vital role in different scenarios like telecommunications, high performance computing and multiprocessor systems. Load balancing is an emerging issue in MINs, surprisingly there is very less work done on this. Fault tolerance, reliability, and load balancing are the parameters to evaluate the performance of MINs. In Load balancing, the workload is distributed across multiple computers, links, or central processing units or other resources, to achieve optimal resource utilization, maximize throughput, minimize response time, and avoid overload or congestion. Congestion is situation, which occurs due the overload on the network. In MIN, the number of packets sent, is greater than the capacity of the switch i.e. the number of packets a switch can handle. In an electrical MIN, link conflicts or congestive collapse may also occur due to congestion. When more than two or more packets from different sources request the same output port in a switch simultaneously then the condition of congestive collapse or link conflict (LC) arises. In other words, when two or more packets try to access the same wavelength at same time, then the problem of congestive collapse or link conflict occurs [23]. To solve the link conflict problem, the time domain approach is used. From previous researches we analyzed that Space domain approach [1], time domain approach [10],[12],[15] and wavelength domain approach[2],[6],[7] are the three approaches which can be used for to avoid conflicts. In Time Domain Approach, the connections are established at different time periods when two or more wavelengths try to access the same wavelength at same time [1],[10],[12],[15]. Some algorithms in which time domain approach is used are Windows Method [1],[5], Improved Windows Method [1], [3], Bitwise Window Method [14-15], Four Heuristic Algorithms [19], Simulated Annealing (SA) [4], and Zero algorithms [3],[8].

\section{PROPOSED APPROACH}

Due to traffic load on a single link, the problem of link conflict can arise. As in previous definitions, when two or more packets from different sources request the same link or wavelength in a switch simultaneously, the congestion occurs at that requested output link (or wavelength) and this leads to link conflict (or collapse congestive). The link conflict in a switch is shown in Fig. 1 (a) and Fig. 1 (b).

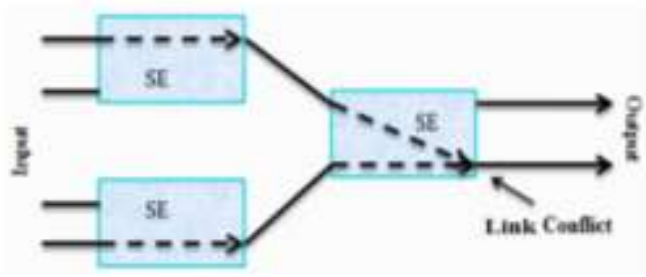

(a)
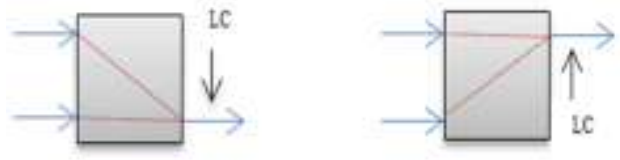

(b)

Figure 1. Link Conflict 
In this paper, we have proposed an algorithm for detecting link conflicts and provide method for resolving these conflicts. In this algorithm, we schedule the messages in different independent subsets in order to avoid the path conflicts in the MINs. Multistage interconnection network (MIN) is a class of high-speed computer networks. An Omega Network, which consists of $\log _{\mathrm{m}} \mathrm{N}$ stages of $\mathrm{m} \times \mathrm{m}$ switching elements (SEs) connecting $\mathrm{N}$ input terminals to $\mathrm{N}$, output terminals. We have taken an $\mathrm{NxN}$ Omega network consists of $2 \times 2$ switching elements. The number of stages is $\log _{2} \mathrm{~N}$ and numbers of switching elements in each stage are $\mathrm{N} / 2$. The arrangement of stages is from 0 to $\left(\log _{2} \mathrm{~N}\right)-1$ in which $\left(\log _{2} \mathrm{~N}\right)-1$ is the last stage. The route selection of each data packet is according to its destination address. An 8 x 8 Omega Network with 3 -stages is shown in Fig. 2 . If some or all the source addresses accesses their destination addresses in a same time slot[23], then there may occurs the problem of link conflict in the omega network, which is represented by dotted lines in fig. 2. and the conflicted routes are represented by arrow. In fig. 2, the link conflicts occurs when signals trying to access the same path (link) at the same time slot (cycle) within the switching element.

\subsection{Link Window Method (LWM)}

The Link Window Method (LWM) is proposed to find link conflicts, so we can schedule messages within same pool according to their conflicts in network [1][15][22]. In Link Window Method (LWM), NxN network consist of $\mathrm{N}$ source and $\mathrm{N}$ destination address. These addresses (source address (SA) and its corresponding destination address (DA)) are combined to formed link combination matrix. Let the link window size is $\mathrm{L}_{\mathrm{s}}=\log _{2} \mathrm{~N}$ and number of link window $L_{w}=S-1$ where $N$ is network size. The size of link window $\left(\mathrm{L}_{\mathrm{s}}\right)$ is same as number of stages of the network. The $\mathrm{L}_{\mathrm{w}}$ is counted from left side in the link combination matrix and the first and last column are not used. There occurs a link conflict, if two messages having same bit pattern in Lw. So they are scheduled to be routed in different passes.

For example, in Link Window Method (LWM), an $8 \times 8$ network size $(\mathrm{NxN})$, link window size $\left(\mathrm{L}_{\mathrm{s}}\right)$ $=$ number of stages $=\log _{2} \mathrm{~N}=3$ and number of link window $\mathrm{L}_{\mathrm{w}}=\mathrm{S}-1=2\left(\mathrm{~L}_{\mathrm{w} 0}, \mathrm{~L}_{\mathrm{w} 1}\right)$. Each Source $(\mathrm{SA})$ and its Destination address (DA) are pooled as shown in Fig. 3. and link conflicts in $8 \times 8$ omega network is shown in Fig. 2.

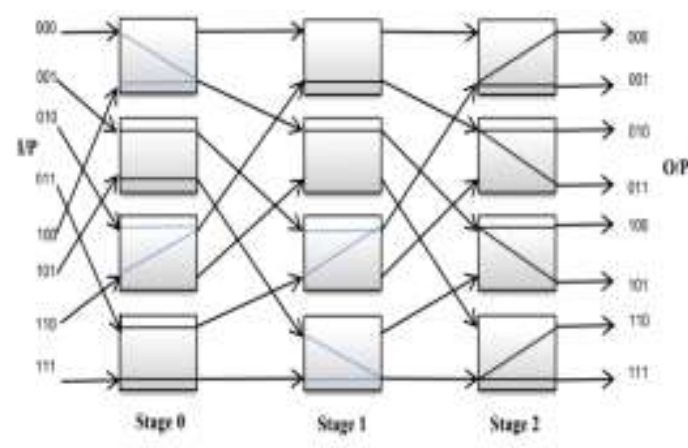

Figure 2. 8x8 Omega Network with Link Conflicts

Fig. 3. shows link combination matrix of SA and DA and Fig. 4. shows link window method. In first Link Window $\left(\mathrm{L}_{\mathrm{w} 0}\right)$, conflicts are between messages (000 \& 100) and (010 \& 110). In second Link Window $\left(\mathrm{L}_{\mathrm{w} 1}\right)$ conflicts are between messages $(000 \&$ 100), (001 \& 011), (101\& \& 111) and (010 \& 110).

\begin{tabular}{|l|}
\hline SA DA \\
000100 \\
001000 \\
010011 \\
011001 \\
100101 \\
101110 \\
110010 \\
111111 \\
\hline
\end{tabular}

Figure 3. Link Combination Matrix

\begin{tabular}{|c:c:cc|c|c|}
\hline & $L_{\mathrm{w}}$ & & \\
\hdashline 0 & 0 & 0 & 0 & 0 \\
0 & 0 & 1 & 0 & 0 & 0 \\
0 & 1 & 0 & 0 & 1 & 1 \\
0 & 1 & 1 & 0 & 0 & 1 \\
1 & 0 & 0 & 1 & 0 & 1 \\
1 & 0 & 1 & 1 & 1 & 0 \\
1 & 1 & 0 & 0 & 1 & 0 \\
1 & 1 & 1 & 1 & 1 & 1 \\
\hline
\end{tabular}

(a) 


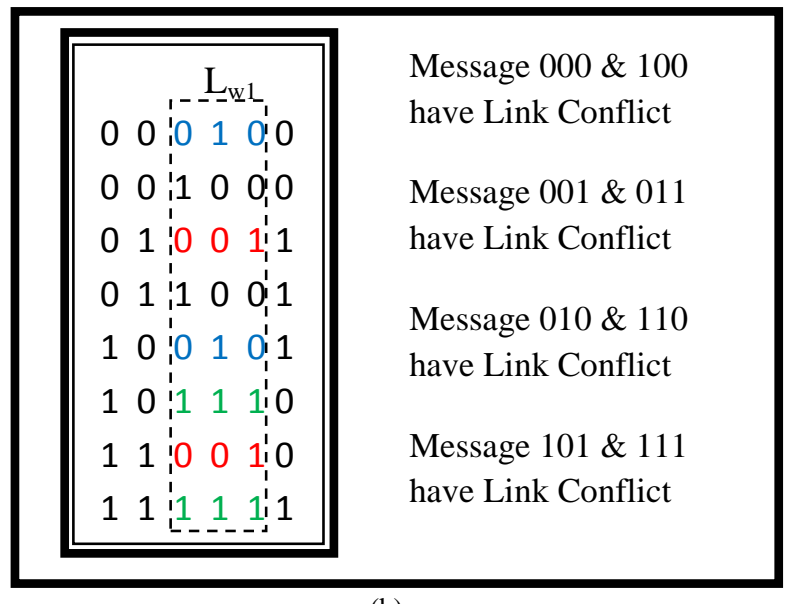

(b)

Figure 4. (a) Link Window $0\left(\mathrm{~L}_{\mathrm{w} 0}\right)$ (b) Link Window 1

$$
\left(\mathrm{L}_{\mathrm{w} 1}\right)
$$

The Pseudo Code for finding Link Conflicts in link window method is as shown below:

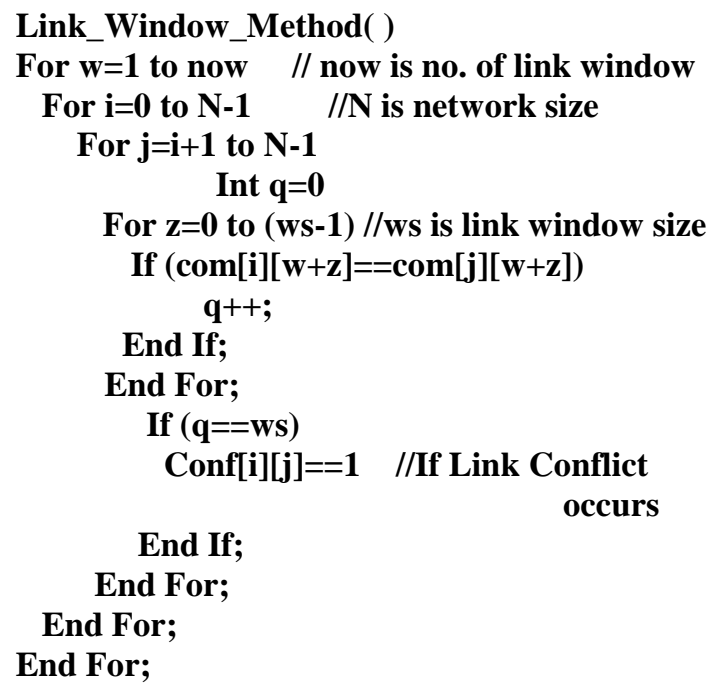

Link Conflict Matrix displays link conflicts, which are shown in below table. If there is a conflict between two messages, it can be represented as 1 otherwise 0 . For example, message $000 \& 100$ have link conflict, the conflict matrix can be assigned as 1 . Therefore, with link window method, we find four link conflicts, which are represented as 1 in link conflict matrix table 1 .
Table I. Link Conflict Matrix

\begin{tabular}{|l|l|l|l|l|l|l|l|l|}
\hline Message & 000 & 001 & 010 & 011 & 100 & 101 & 110 & 111 \\
\hline 000 & 0 & 0 & 0 & 0 & 1 & 0 & 0 & 0 \\
\hline 001 & 0 & 0 & 0 & 1 & 0 & 0 & 0 & 0 \\
\hline 010 & 0 & 0 & 0 & 0 & 0 & 0 & 1 & 0 \\
\hline 011 & 0 & 0 & 0 & 0 & 0 & 0 & 0 & 0 \\
\hline 100 & 0 & 0 & 0 & 0 & 0 & 0 & 0 & 0 \\
\hline 101 & 0 & 0 & 0 & 0 & 0 & 0 & 0 & 1 \\
\hline 110 & 0 & 0 & 0 & 0 & 0 & 0 & 0 & 0 \\
\hline 111 & 0 & 0 & 0 & 0 & 0 & 0 & 0 & 0 \\
\hline
\end{tabular}

Thus Link conflicts (or congestive collapse) in omega network can be shown in Link conflict graph as shown in Fig. 5 and are represented as yellow lines.

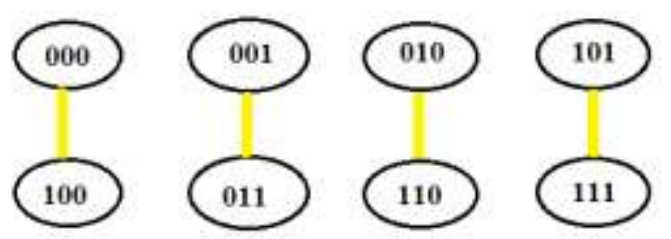

Figure 5. Link Conflict Graph

When link conflict (or congestive collapse) is detected between two messages, then we can put them in different pools. With time domain approach these messages are routed in different passes (or time slot). Therefore, messages 000, 001, 010, and 101 are put into one pool and can be routed in same time slot because they do not have link conflict (or congestive collapse) with each other. Similarly messages 100 , $011,110,111$ are routed in another time slot.

\section{RESULTS AND DISCUSSION}

The Link Window (LWM) method is compared with the existing Windows method (WM) and Improved Windows method (IWM) for different network sizes. This comparison shows that the time spent for detecting and identifying conflicts in Link Window method (LWM) is very less as compared to Windows Method (WM) and Improved Windows Method (IWM) which is mentioned in Table 2.

Table II. Comparison of LWM, WM, and IWM

\begin{tabular}{|c|c|c|c|}
\hline $\begin{array}{c}\text { Network } \\
\text { Size }\end{array}$ & $\begin{array}{c}\text { LWM } \\
\text { (nanosec) }\end{array}$ & $\begin{array}{c}\text { WM } \\
\text { (nanosec) }\end{array}$ & $\begin{array}{c}\text { IWM } \\
\text { (nanosec) }\end{array}$ \\
\hline $\mathbf{4}$ & 5802 & 7588 & 6695 \\
\hline $\mathbf{8}$ & 26780 & 28118 & 39276 \\
\hline $\mathbf{1 6}$ & 146842 & 162464 & 191921 \\
\hline $\mathbf{3 2}$ & 7301919 & 7500980 & 1283638 \\
\hline $\mathbf{6 4}$ & 5009581 & 5787975 & 7642020 \\
\hline
\end{tabular}

\section{CONCLUSION}

Therefore, in omega network traffic load or congestion can be balanced in switches with the help of proposed algorithm. This approach is not only 
resolving link conflicts but also provide electrical crosstalk-free routes. This Link Window Method (LWM) can be also applied in optical MIN to detect link conflicts. In this paper, the exact position of the link conflicts as well as congestion between source and destination address can be find easily. The LWM improves the performance of finding link conflicts in multistage network. Therefore, with the Link window method and time domain approach, messages can be routed conflict free. In future, we will work on Optical switch and link conflicts and minimize the execution time of routing.

\section{ACKNOWLEDGEMENTS}

The author would like to thank her family and guide who have been a pillar of energy for her.

\section{REFERENCES}

[1] Shen, X., Yang, F., and Pan, Y., Equivalent permutation capabilities between time division optical omega networks and non-optical extra-stage omega networks, IEEE/ACM Trans. Netw. Vol. 9, No. 4, pp. 518 -524, 2001.

[2] M.A.Al-Shabi, ZeroX Algorithms with Free crosstalk in Optical Multistage Interconnection Network, International Journal of Advanced Computer Science and Applications (IJACSA), Vol. 4, No. 2, 2013.

[3] M.A. Al-Shabi and M. Othman, A new algorithm for routing and scheduling in optical omega network, International Journal of The Computer, the Internet and Management, 16(1), pp. 26-31, 2008

[4] K. Padmanabhan and A. N. Netravali, Dilated Networks for Photonic Switching, IEEE Transactions on Communications, Vol. 35, No. 12, pp. 1357-1365, 1987.

[5] Munir, A, Mohamed, O., and Rozita, J., An efficient approach to avoid crosstalk in optical Omega Network, International Journal of Computer, Internet and management, Vol.14, No.1, pp.50-60, 2005.

[6] E. Lu and S. Q. Zheng, Parallel Routing and Wavelength Assignment for Optical Multistage Interconnection Networks, in: Proceedings of the International Conference on Parallel Processing (ICPP'04), 2004.

[7] A.S. Almazyad, Optical omega networks with centralized buffering and wavelength conversion, Journal of King Saud University - Computer and Information Sciences, vol. 23 , no. 1, pp. 15-28, 2010.

[8] Shahida T. D., Othman M. \& Khazani M., Fast ZeroY Algorithm for Efficient Message Routing in Optical Multistage Interconnection Networks, Proceedings of the 3rd International Symposium on Information Technology 2008 (ITSim’08),pp. 2369-2374, 2008.

[9] G. Shanmugam, P. Ganesan and P.T. Vanathi, Meta heuristic algorithms for vehicle routing problem with stochastic demands, J. Comput. Sci., vol. 7, pp. 533542,2011
[10] R. Aggarwal, L. Kaur and H. Aggarwal, Multistage interconnection networks: a transition from electronic to optical, Journal of Emerging Technologies in Web Intelligence, 2(2), pp.142-147, 2010.

[11] Mehrnaz Moudi and Mohamed Othman, A Cost-Effective Architecture For Optical Multistage Interconnetion netowrk, Journal of networks, Vol. 8, No. 2, February 2013.

[12] F. Abed and M. Othman, "Fast method to find conflicts in optical multistage interconnection networks", International Journal of The Computer, The Internet and Management, 16(1), pp. 18-25, 2008

[13] M. Abdullah, M. Othman and R. Johari, An efficient approach for message routing in optical omega network, International Journal of The Computer, the Internet and Management, 14(1), pp. 50- 60, 2006.

[14] S. Kaur, Anantdeep and D. Aggarwal, Effect of crosstalk on permutation in optical multistage interconnection networks, Journal of Computing, 2(4), pp. 100-104, 2010.

[15] Y. Yang, J. Wang and Y. Pan, Permutation capability of optical multistage interconnection networks, Journal of Parallel and Distributed Computing, 60, pp. 72-91, 2000.

[16] P.K. Bansal, R.C. Joshi, K. Singh, On a fault-tolerant multistage interconnection network, Journal of Computers and Electrical Engineering, Elsevier Sciences 20 (4), pp. 335-345, 1994.

[17] J. Duato, S. Yalamanchili, L. M. Ni, Interconnection Networks: An Engineering Approach, Morgan Kaufmann, San Francisco, CA, 2003, ISBN 1-55860-852-4.

[18] T. W. Manikas and J. T. Cain, Genetic Algorithms vs. Simulated Annealing: A Comparison of Approaches for Solving the Circuit Partitioning Problem, Technical Report 96-101, Department of Electrical Engineering, University of Pittsburgh, 1996.

[19] A. K. Katangur, S. Akkaladevi, Y. Pan and M. D. Fraser, Applying Ant Colony Optimization to Routing Optical Multistage Interconnection Networks with Limited Crosstalk, Proceedings of the 18th International Parallel and Distributed Processing Symposium, pp. 163-170, 2004.

[20] S. C. Chau, and T. Xiao, A New Algorithm for Routing and Scheduling in Optical Multistage Interconnection Networks, Proceedings of the 4th IASTED International Multi Conference on Wireless and Optical Communications, Banff, Canada, pp. 749-755, 2004.

[21] D. Shahida, M. Othman and M. Khazani, Routing Algorithms in Optical Multistage Interconnection Networks: Revisited, Proceedings of the 3rd World Engineering Congress, pp. 63-70, 2007.

[22] Pan, Y. Qiao, C. and Yang, Y. Optical Multistage Interconnection Networks: New Challenges and Approaches, IEEE Communications Magazine, Feature Topic on Optical Networks, Communication System, 1999.

[23] Shobha Arya, Nipur, Link and Switch Conflicts-Free Routing in Optical Multistage Interconnection Networks", Proceeding of 1st International Conference on Next Generation Computing Technologies (NGCT2015), September 2015.

[24] C. G'omez, F. Gilabert, M.E. G'omez, P. L'opez, J. Duato, Beyond Fat-tree: Unidirectional Load-Balanced Multistage Interconnection Network, IEEE Computer Architecture Letters, Vol 7, No. 2, July-December 2008. 